\title{
ORIGINAL
}

\section{PREVALENCIA DE LA DISCAPACIDAD EN ESPAÑA POR COMUNIDADES AUTÓNOMAS: EL PAPEL DE LOS FACTORES INDIVIDUALES Y DEL ENTORNO GEOGRÁFICO EN SU VARIABILIDAD}

\author{
(1) Servei d'Informació i Estudis. Departament de Salut. \\ (2) Universitat Politècnica de Catalunya. \\ (3) Universitat de Lleida. \\ (4) Institut Català de la Salut. \\ (5) Consejería de Salud. Junta de Andalucía. \\ (6) Fundació Institut Català de l’Envelliment.
}

Rosa Gispert Magarolas (1), Guillem Clot-Razquin G (1, 2), Jaume March Llanes (3), Adriana Freitas Ramírez (1), Ester Busquets Bou (4), Miguel Ruíz-Ramos (5) y Antoni Rivero Fernández (6).

\section{RESUMEN}

Fundamento: La prevalencia de discapacidad en la población general presenta una gran variabilidad geográfica, de manera que identificar aquellos factores que pudieran explicarla será importante para la planificación de políticas sociales. En este trabajo se analiza la variabilidad de la discapacidad por comunidades autónomas desde una doble vertiente, los factores individuales y del entorno.

Métodos: Los datos proceden principalmente de la Encuesta de Discapacidad, Deficiencias y Estado de Salud de 1999 y del Inebase, ambas del Instituto Nacional de Estadística (INE). Se calculó la prevalencia de discapacidad simple y ajustada por edad de las CCAA. Se analizan los factores individuales asociados a la discapacidad mediante una regresión logística y los factores individuales y de la comunidad autónoma conjuntamente con una regresión logística de dos niveles.

Resultados: La prevalencia de discapacidad muestra una diferencia máxima de 5,75 puntos entre las comunidades autónomas. En la regresión logística la comunidad de residencia fue estadísticamente significativa (OR: 3,35 en la de mayor prevalencia respecto a la de menor) junto con otras variables individuales: edad (OR de $40-64=1,78$ OR de $65-79=1,87$ y OR de $>79=3,34$ ), sexo (OR mujer $=0,66)$, situación laboral $(\mathrm{OR}$ sin trabajo $=2,25 \mathrm{OR}$ amas casa/estudiante $=1,39$ y OR otros $=2,03$ ), estado de salud (OR regular $=1,69 \mathrm{OR}$ malo/muy malo=2,05) y enfermedades crónicas $(\mathrm{OR}$ $1-3=1,56$ OR $4-6=1,82$ OR $>6=2,59)$. En la regresión de dos niveles las variables individuales explican poca varianza $(\sigma=0,261)$ y ninguna de las variables relativas a las CCAA mejora el modelo.

Conclusiones: Las características individuales no explican suficientemente la variabilidad de la discapacidad entre CCAA y no se han identificado variables del entorno que sean significativas.

Palabras clave: Personas discapacitadas. Areas pequeñas. Análisis multinivel. España.

\section{Correspondencia:}

Rosa Gispert

Servei d'Informació i Estudis. Departament de Salut

C/ Travessera de les Corts 131-159. 08028 Barcelona

Correo electrónico: rosa.gispert@gencat.cat

Financiación: FIS PI052650

Conflictos de intereses: ninguno

\section{ABSTRACT \\ Prevalence of the Disability in Spain for Autonomous Communities: the Paper of the Individual Factors and the Geographical Environment in the Variability}

Background: The prevalence of disability shows a high geographical variability and the identification of factors that could explain these variations can be usuful to the heath and social welfare planning. Here the analysis of disability variations among autonomous regions in Spain is made taking into account individual and geographical setting factors together.

Methods: Data come from the Spanish Disability, Impairment and Health Status survey of 1999 and from Inebase both of the National Institute for Statistics. The prevalence of disability crude and adjusted by age was calculated for each autonomous region. Individual factors related to disability are identified by means of a logistic regression. The analysis accounting for both, individual and geographical setting factors was performed by means of a logistic regression of two levels.

Results: Disability prevalence showed a maximum difference of 5.75 points among regions. In a logistic regression the region of residence was statistically significant (OR: 3.35 in the highest rated region related to the lowest) beside several individual factors: age (OR 40-64=1,78 OR 65-79=1,87 and OR $>79=3,34)$, sex $(\mathrm{OR}$ women $=0,66)$, working status $(\mathrm{OR}$ unemployment $=2,25 \mathrm{OR}$ housewife/student $=1,39$ y OR other $=2,03$ ), health status (OR regular $=1,69 \mathrm{OR}$ bad/very bad $=2,05)$ and chronic diseases $(\mathrm{OR}$ $1-3=1,56$ OR $4-6=1,82$ OR $>6=2,59$ ). Individual factors accounted for a very few variance at the two levels regression model $(\sigma=0,261)$ and none of the regional variables improved the model.

Conclusions: Individual factors do not explain enough the observed disability variations among the regions and none factor related to the geographical setting has been identified as statistically significant.

Key words: Disabled persons. Small areas analysis. Multilevel analysis. Spain. 


\section{INTRODUCCIÓN}

Con mucha frecuencia la discapacidad es una condición que afecta a la salud de las personas, y por este motivo se asocia a muchos de los llamados factores determinantes de la salud. La edad, el sexo, la situación laboral así como otros factores sociales y demográficos condicionan en gran medida tanto la forma de enfermar como la de enfrentarse a las consecuencias de la enfermedad $^{1-3}$.

En el ámbito clínico es habitual trabajar con escalas de medida para evaluar la capacidad funcional y la dependencia, las cuales han sido diseñadas ad-hoc y validadas previamente en el contexto asistencial, de forma que en su implementación ya se tienen en cuenta algunos de estos factores condicionantes ${ }^{4}$. En el caso de la información poblacional, que se utiliza fundamentalmente para la elaboración de indicadores de referencia para el conjunto de la población, las posibilidades de ajuste por este tipo de variables no es tan frecuente ${ }^{5}$. Sin embargo, la posibilidad de diferenciar distintos grados de discapacidad en base a su gravedad, comorbilidad o niveles de limitación funcional, permite ajustar las estimaciones de la población en situación de dependencia ${ }^{6}$.

Por otra parte, algunos estudios han puesto de manifiesto importantes diferencias en la distribución geográfica de la prevalencia de discapacidad a lo ancho del territorio español y más concretamente por comunidades autónomas $(\mathrm{CCAA})^{7}$. Una distribución distinta en el territorio de algunos de estos factores podría justificar las diferencias en la discapacidad e incluso sugerir medidas para reducirlas. Por este motivo, analizar esta variabilidad y los factores que pueden explicarla puede ser de gran utilidad, tanto para entender la naturaleza de este problema de salud como por sus repercusiones en la planificación y la asignación de recursos ${ }^{8}$. Además puede ayudar también a comprender la variabilidad observada en otros indicadores basados en parte en los datos de discapacidad, como son la dependencia y la esperanza de vida libre de incapacidad, los cuales se utilizan para monitorizar la salud de la población ${ }^{8,9}$.

Los datos disponibles de la encuesta de discapacidad del $\mathrm{INE}^{7}$ pueden ser de gran utilidad para analizar estas diferencias, en primer lugar por la magnitud de la muestra $\mathrm{y}$ en segundo por el hecho de incorporar características sociosanitarias del individuo y de su entorno.

El objetivo de este trabajo ha sido explorar la variabilidad geográfica de la prevalencia de discapacidad entre las comunidades autónomas y los factores que pueden explicarla desde una doble vertiente, por un lado desde la perspectiva individual y por otro a partir de un diseño de estudio ecológico usando como unidad de análisis la comunidad autónoma de residencia.

\section{MATERIAL Y MÉTODOS}

Los datos utilizados fundamentalmente proceden de la encuesta de discapacidad deficiencias y estado de salud (EDDES) de 1999 del Instituto Nacional de Estadísti$\mathrm{ca}^{7}$. Esta encuesta se compone de diversos cuestionarios y muestras de las que en este trabajo hemos utilizado dos. La primera muestra (que denominamos tododis) corresponde a las entrevistas realizadas en todos los hogares seleccionados para detectar a las personas que presentan alguna discapacidad a partir de una pregunta filtro, y a las que se administraba el cuestionario de discapacidad. De ella se extrajeron los datos para el cálculo de la prevalencia poblacional de discapacidad por comunidad autónoma. La segunda muestra (que denominamos saludis) está formada 
por personas seleccionadas aleatoriamente en cada hogar y que cumplimentaron un cuestionario de salud. Comprende datos sociodemográficos, de hábitos, relación con el entorno y de estado de salud. Una parte de las personas que respondieron al cuestionario de salud presentaban discapacidades (es decir que respondieron a ambos cuestionarios).

También se utilizaron datos sociodemográficos, económicos y de servicios sanitarios de las CCAA procedentes de las bases de datos estadísticas disponibles para cada una de ellas, principalmente del $\mathrm{INE}^{10}$ (censo de población y vivienda, encuesta de población activa, padrón municipal, encuesta de condiciones de vida y estadística de indicadores hospitalarios y del IMSERSO $^{11}$ (plazas de larga estancia y residenciales). De ellas se obtuvieron 14 variables (\% casados, \% familias monoparentales, \% hogares unipersonales, $\%$ un núcleo solo, tasa de paro, \% industria, renta disponible bruta, coches matriculados, \% hogares de propiedad, $\%$ mayores de 65 años, \% municipios $<10.000$ habitantes, \% agricultura, densidad población, plazas sociosanitarias), que se utilizaron en un análisis exploratorio de componentes principales para caracterizar a las CCAA (no presentado en este artículo) cuyos resultados se emplearon en el presente trabajo.

Se ha calculado la prevalencia de discapacidad por CCAA (a partir del lugar de residencia del entrevistado), edad y sexo utilizando las dos muestras y se compararon sus resultados para comprobar la coherencia entre esas dos fuentes. En ambos casos se ponderó la muestra. Se calculó el intervalo de confianza para el estimador de prevalencia de la muestra de salud ya que se asume que la estimación más correcta metodológicamente sería la realizada con la muestra de discapacidad. La prevalencia calculada con la muestra tododis que se presenta, además se ha estandarizado con el método directo por edad y sexo, usando como estándar la población española de ambos sexos del censo de $2001^{10}$.

Para identificar los factores individuales asociados a la discapacidad se realizó una regresión logística, utilizando los datos procedentes del cuestionario de salud (saludis), siendo la variable dependiente tener o no alguna discapacidad y como variables explicativas las características sociales, demográficas, etc. recogidas por esta encuesta. Para realizar esta regresión se ha utilizado el programa SPSS.

Para analizar conjuntamente los factores individuales y de grupo (el entorno) se realizó una regresión logística de dos niveles. El primer nivel correspondió a los individuos y el segundo a la comunidad autónoma. Los datos y las variables utilizadas a nivel individual fueron las mismas que en la regresión logística previa, excluyendo la comunidad de residencia de cada individuo. En el segundo nivel se utilizaron variables descriptivas de la Comunidad Autónoma seleccionadas a partir de un análisis de componentes principales previo. Este análisis permitió seleccionar unas pocas variables representativas de cada uno de los cuatro grandes grupos identificados (Familia, Renta, Entorno y Servicios). Para la regresión con dos niveles se supuso que las pendientes son iguales en las diferentes CCAA, ya que de otra forma el modelo se complicaría demasiado y dificultaría su interpretación. Se modeló la constante como efecto aleatorio a nivel de comunidad autónoma. Para reducir la variabilidad que hay entre comunidades autónomas se modeló como efectos fijos las siete variables de segundo nivel. Para este análisis se utilizó la función glmmPQL de la librería MASS del programa R 2.4.1 ${ }^{12}$. Esta función estima los parámetros por el método del PQL (Penalized Quasi-Likelihood). 


\section{RESULTADOS}

El número de personas incluidas en el estudio fue de 218.185 de las cuales 20.224 presentaron alguna discapacidad y respondieron al cuestionario de discapacidad (tododis). Por otra parte, 67.051 personas seleccionadas al azar de los hogares visitados respondieron al cuestionario de salud (saludis), de ellas 9.029 presentaban alguna discapacidad y también habían contestado el primer cuestionario.

La prevalencia de discapacidad para el conjunto de la población española de los dos sexos se situó en 8,99 por cada 100 habitantes, siendo de 7,66 en hombres y de 10,27 en mujeres. Se observó un rango de 5,75 puntos entre las CCAA con la mayor y la menor frecuencia para el conjunto de los dos sexos (Castilla León 11,35 \% y La Rioja 5,6\% respectivamente). La diferencia entre hombres y mujeres se apreció en todas las CCAA, siendo Baleares la que mostró menor diferencia ( Hombres: 7,19\% y Mujeres: $8,07 \%$ ) y Ceuta y Melilla la que más (Hombres: 6,92 y Mujeres: 11,43) (tabla 1 y figura 1 ).

La prevalencia estandarizada por edad fue de $8,79 \%$ en los hombres, de 9,65\% en las mujeres y de $9,31 \%$ en el total (tabla 1). Al estandarizar por edad las diferencias entre los dos sexos con respecto a los valores no estandarizados disminuyeron, siendo en general inferiores en las mujeres y superiores en los hombres. En tres CCAA después de estandarizar, la prevalencia en los hombres superó la de las mujeres (Asturias 10,98\% y $9,80 \%$, Baleares $8,01 \%$ y $7,62 \%$, La Rioja $7,43 \%$ y $5,91 \%$ respectivamente) y el rango de la diferencias entre sexos se redujo, siendo Galícia la que mostró menor diferencia (Hombres: $10,73 \%$ y

Tabla 1

Prevalencia (en \%) de discapacidad según Comunidad Autónoma y sexo

\begin{tabular}{|c|c|c|c|c|c|c|}
\hline & \multicolumn{3}{|c|}{ Prevalencia } & \multicolumn{3}{|c|}{ Prevalencia Estandarizada } \\
\hline & Varón & Mujer & Total & Varón & Mujer & Total \\
\hline Andalucía & 8,71 & 11,45 & 10,10 & 10,09 & 10,85 & 10,51 \\
\hline Aragón & 7,49 & 9,27 & 8,39 & 8,55 & 8,87 & 8,71 \\
\hline Asturias & 9,25 & 10,16 & 9,72 & 10,98 & 9,80 & 10,35 \\
\hline Baleares & 7,19 & 8,07 & 7,64 & 8,01 & 7,62 & 7,77 \\
\hline Canarias & 5,78 & 7,52 & 6,66 & 6,37 & 7,03 & 6,74 \\
\hline Cantabria & 8,13 & 10,57 & 9,37 & 9,54 & 9,91 & 9,86 \\
\hline Castilla-La Mancha & 8,43 & 11,37 & 9,91 & 10,04 & 10,97 & 10,33 \\
\hline Castilla-León & 9,75 & 12,91 & 11,35 & 11,58 & 12,56 & 12,19 \\
\hline Cataluña & 7,96 & 10,81 & 9,41 & 8,98 & 10,18 & 9,72 \\
\hline Ceuta y Melilla & 6,92 & 11,43 & 9,13 & 7,98 & 9,96 & 8,77 \\
\hline Extremadura & 7,77 & 11,50 & 9,65 & 10,16 & 10,97 & 10,27 \\
\hline Galicia & 8,93 & 11,47 & 10,24 & 10,73 & 10,9 & 10,83 \\
\hline La Rioja & 5,01 & 6,18 & 5,60 & 7,43 & 5,91 & 6,01 \\
\hline Madrid & 5,57 & 8,34 & 7,01 & 6,18 & 7,56 & 7,04 \\
\hline Murcia & 9,08 & 11,55 & 10,33 & 10,01 & 10,83 & 10,51 \\
\hline Navarra & 7,04 & 8,38 & 7,71 & 8,13 & 8,50 & 8,39 \\
\hline País Vasco & 5,88 & 8,38 & 7,16 & 6,81 & 8,22 & 7,64 \\
\hline Valencia & 6,65 & 9,44 & 8,08 & 7,66 & 8,93 & 8,38 \\
\hline España & 7,66 & 10,27 & 8,99 & 8,79 & 9,65 & 9,31 \\
\hline
\end{tabular}


Figura 1

Prevalencia (en \%) de discapacidad por Comunidad Autónoma y sexo

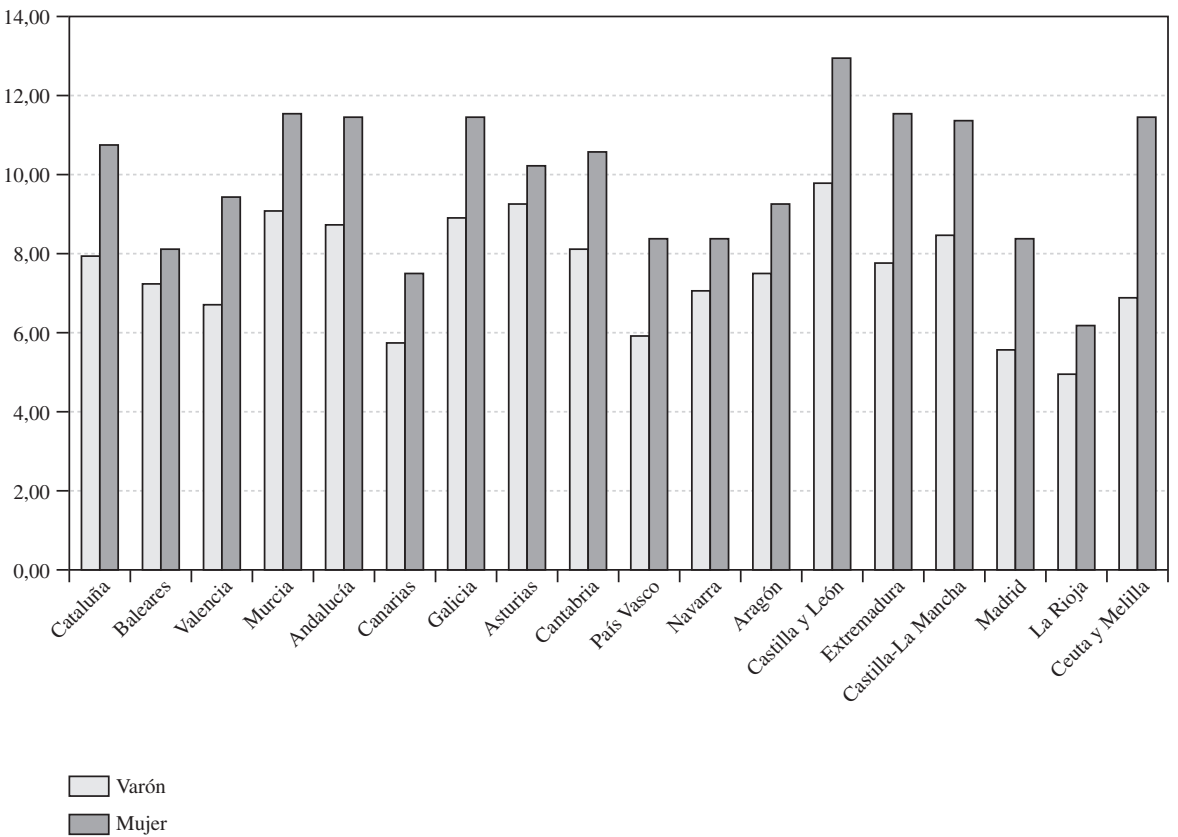

Mujeres: $10,90 \%)$ y Ceuta y Melilla la mayor (Hombres:7,98\% y Mujeres: 9,96\%). A pesar de estandarizar por edad, la variabilidad en la distribución territorial apenas se modificó $(\sigma=1,65)$. En la figura 2 se muestran los mapas con la distribución de la prevalencia sin estandarizar y estandarizadas respectivamente. Se puede constatar que la variabilidad permaneció y el patrón territorial fue parecido, aunque al estandarizar la prevalencia aumentó en la mayoría de CCAA y alguna de ellas cambió a la categoría superior de valores (Asturias, Cantabria, País Vasco, Aragón, Extremadura).

En la figura 3 se presentan los resultados del cálculo de la prevalencia de discapacidad por Comunidad Autónoma según las dos fuentes de datos. El punto representado por una estrella oscura en la figura indica la estimación a partir de la mues- tra de discapacidad (tododis), el punto claro la estimación según la muestra del cuestionario de salud (saludis) y las líneas su intervalo del $95 \%$ de confianza. Podemos observar que en todas las CCAA excepto Aragón, el intervalo incluía el valor. A partir de esta comprobación se consideró que la utilización de los datos de la muestra de población que respondió al cuestionario de salud (saludis) sería aceptable para la estimación de la prevalencia de discapacidad $\mathrm{y}$, fundamentalmente, para el estudio de los factores individuales asociados, objetivo principal del estudio.

Mediante la regresión logística se identificaron las variables individuales asociadas a presentar al menos una discapacidad (datos de saludis) en la población general. En la tabla 2 se incluyen las variables que muestran significación estadística en 
Figura 2

Prevalencia de discapacidad por Comunidad Autónoma sin estandarizar y estandarizada por edad. Ambos sexos
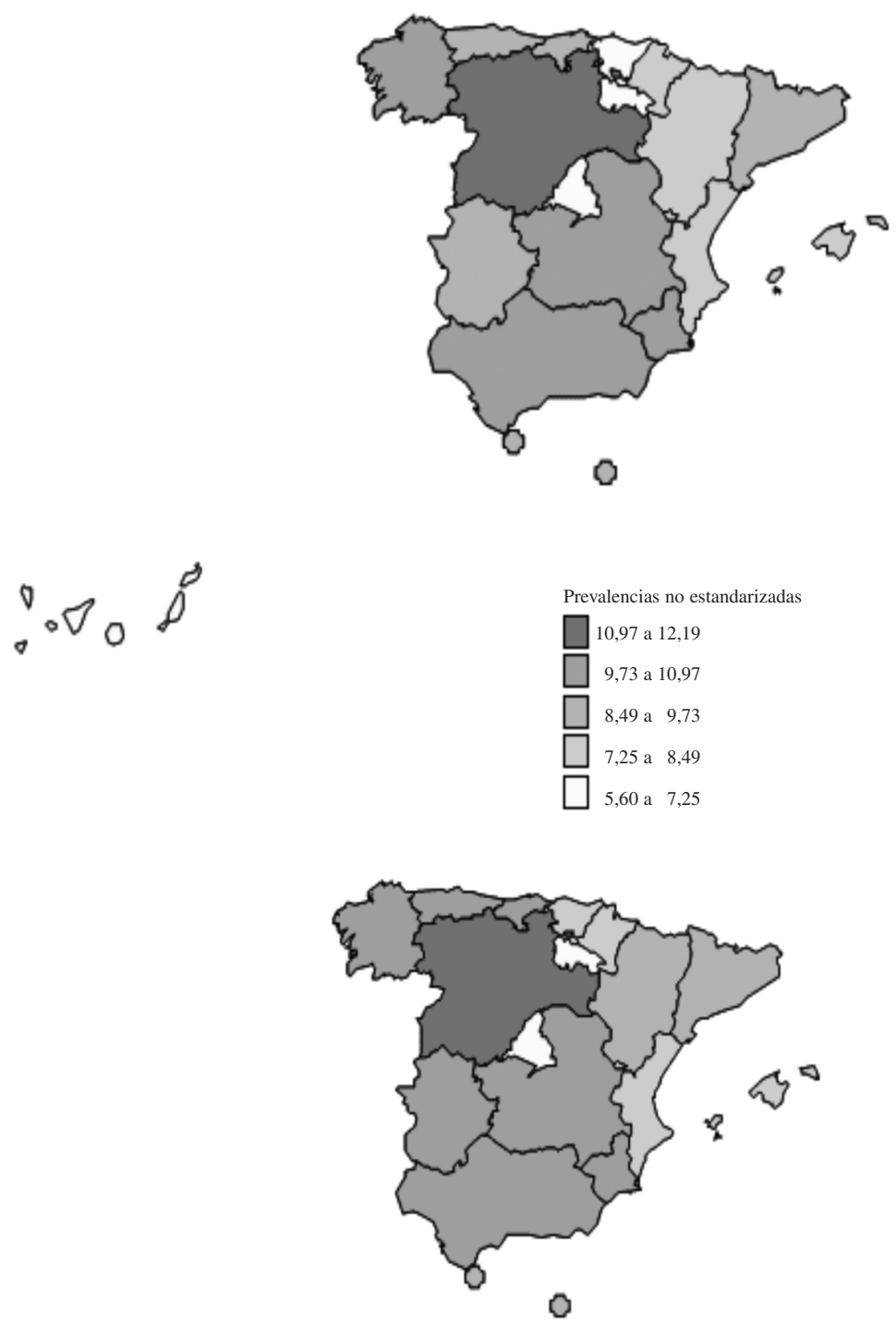

0.00

Prevalencias estandarizadas
$\square$ 10,97 a 12,19
$\square, 73$ a 10,97
$\square, 49$ a 9,73
$\square \quad 7,25$ a 8,49
$\square, 60$ a 7,25 
Figura 3

Estimación de la prevalencia de discapacidad por CCAA según las dos fuentes de datos

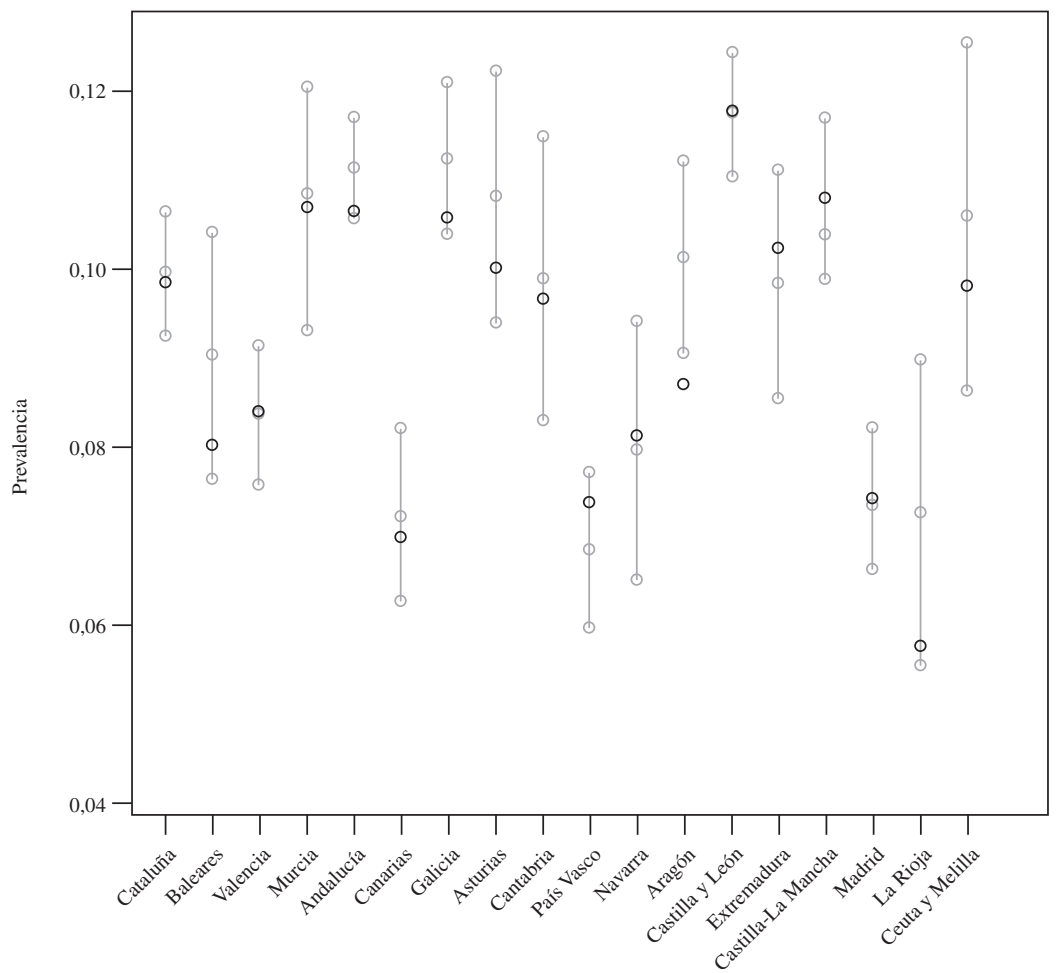

Puntos claro y líneas: estimación e intervalo de confianza con la muestra "saludis".

Punto oscuro: estimación con la muestra "tododis".

alguna de sus categorías (categoría de referencia=1). Destacó una mayor discapacidad asociada con la edad (OR de 40$64=1,78$ OR de $65-79=1,87$ y OR de $>79=3,34)$, el sexo masculino (OR mujer $=0,66)$, la situación laboral $(\mathrm{OR} \sin$ trabajo $=2,25$ OR amas casa/estudiante $=1,39$ y OR otros $=2,03$ ), la percepción del estado de salud (OR regular= 1,69 OR malo/muy malo $=2,05)$ y un número creciente de enfermedades crónicas (OR 1$3=1,56$ OR4-6=1,82 OR>6=2,59). También se asociaron a la discapacidad situaciones relacionadas con el entorno, como las dificultades en la vivienda, el uso del coche y los transportes públicos y el lugar de residencia, con un menor riesgo en las ciudades con más de 500.000 habitantes. A pesar de ajustar por las variables individuales la comunidad autónoma de residencia tuvo diferencias estadísticamente significativas, con un riesgo tres veces mayor $(\mathrm{OR}=3,35)$ entre la comunidad con mayor prevalencia (Castilla-León) respecto de la de menor ( $\mathrm{La}$ Rioja).

Para el análisis de regresión logística de dos niveles se utilizaron los mismos datos individuales de la tabla 2 , excluyendo la comunidad autónoma de residencia en el primer nivel. Puesto que los estadís- 
Tabla 2

Variables individuales asociadas a la discapacidad*

\begin{tabular}{|c|c|c|c|c|c|c|c|c|}
\hline & \multirow{2}{*}{$\mathbf{B}$} & \multirow{2}{*}{ ET } & \multirow{2}{*}{ Wald } & \multirow{2}{*}{ gl } & \multirow{2}{*}{ Sig } & \multirow{2}{*}{ OR } & \multicolumn{2}{|c|}{ IC $95,0 \%$ OR } \\
\hline & & & & & & & Inferior & Superior \\
\hline \multicolumn{9}{|l|}{ Sexo } \\
\hline Varón & & & & & & 1 & & \\
\hline Mujer & $-0,417$ & 0,042 & 97,259 & 1 & 0,000 & 0,659 & 0,606 & 0,716 \\
\hline \multicolumn{9}{|c|}{ Tamaño del municipio de residencia } \\
\hline$<10.000$ hab. & & & 26,853 & 3 & 0,000 & 1 & & \\
\hline $10.001-50.000$ hab. & $-0,072$ & 0,049 & 2,184 & 1 & 0,139 & 0,930 & 0,845 & 1,024 \\
\hline $50.001-500.000$ hab. & $-0,006$ & 0,045 & 0,018 & 1 & 0,892 & 0,994 & 0,911 & 1,085 \\
\hline$>500.000$ hab. & $-0,349$ & 0,072 & 23,212 & 1 & 0,000 & 0,705 & 0,612 & 0,813 \\
\hline \multicolumn{9}{|c|}{ Consumo de medicamentos en los últimos 14 días } \\
\hline No & & & & & & 1 & & \\
\hline Sí & 0,255 & 0,048 & 28,860 & 1 & 0,000 & 1,291 & 1,176 & 1,417 \\
\hline \multicolumn{9}{|l|}{ Dificultades en vivienda } \\
\hline No & & & & & & 1 & & \\
\hline $\mathrm{Si}$ & 0,731 & 0,047 & 240,330 & 1 & 0,000 & 2,077 & 1,893 & 2,278 \\
\hline \multicolumn{9}{|l|}{ Dificultades en coche } \\
\hline No & & & & & & 1 & & \\
\hline $\mathrm{Si}$ & 2,028 & 0,044 & 2127,606 & 1 & 0,000 & 7,602 & 6,974 & 8,286 \\
\hline \multicolumn{9}{|c|}{ Dificultades en transporte público } \\
\hline No & & & & & & 1 & & \\
\hline $\mathrm{Si}$ & 2,232 & 0,053 & 1758,936 & 1 & 0,000 & 9,315 & 8,392 & 10,339 \\
\hline \multicolumn{9}{|l|}{ Nivel de estudios } \\
\hline Superiores & & & 89,346 & 3 & 0,000 & 1 & & \\
\hline Sin estudios & 0,773 & 0,093 & 69,030 & 1 & 0,000 & 2,166 & 1,805 & 2,599 \\
\hline Básicos & 0,565 & 0,090 & 39,101 & 1 & 0,000 & 1,759 & 1,473 & 2,099 \\
\hline Medios & 0,384 & 0,094 & 16,650 & 1 & 0,000 & 1,469 & 1,221 & 1,767 \\
\hline \multicolumn{9}{|l|}{ Actividad laboral } \\
\hline Trabajando & & & 199,086 & 3 & 0,000 & 1 & & \\
\hline Sin trabajo & 0,813 & 0,065 & 155,564 & 1 & 0,000 & 2,254 & 1,984 & 2,561 \\
\hline Ama de casa/estudiante & 0,331 & 0,072 & 21,421 & 1 & 0,000 & 1,393 & 1,210 & 1,602 \\
\hline Otros & 0,711 & 0,094 & 57,475 & 1 & 0,000 & 2,036 & 1,694 & 2,446 \\
\hline \multicolumn{9}{|l|}{ Composición hogar } \\
\hline $1-2$ & & & & & & 1 & & \\
\hline$>2$ & $-0,185$ & 0,048 & 14,921 & 1 & 0,000 & 0,831 & 0,756 & 0,913 \\
\hline \multicolumn{9}{|c|}{ Estado de salud percibido } \\
\hline Bueno/Muy Bueno & & & 198,971 & 2 & 0,000 & 1 & & \\
\hline Regular & 0,529 & 0,041 & 163,246 & 1 & 0,000 & 1,697 & 1,564 & 1,840 \\
\hline Malo/Muy malo & 0,718 & 0,063 & 130,216 & 1 & 0,000 & 2,050 & 1,812 & 2,319 \\
\hline \multicolumn{9}{|l|}{ Enfermedades crónicas } \\
\hline Ninguna & & & 115,515 & 3 & 0,000 & 1 & & \\
\hline $1-3$ & 0,449 & 0,052 & 73,347 & 1 & 0,000 & 1,567 & 1,414 & 1,736 \\
\hline $4-6$ & 0,599 & 0,066 & 82,405 & 1 & 0,000 & 1,820 & 1,599 & 2,071 \\
\hline$>6$ & 0,952 & 0,105 & 81,933 & 1 & 0,000 & 2,590 & 2,108 & 3,183 \\
\hline \multicolumn{9}{|l|}{ Edad } \\
\hline $6-39$ & & & 204,299 & 3 & 0,000 & 1 & & \\
\hline $40-64$ & 0,576 & 0,069 & 69,420 & 1 & 0,000 & 1,780 & 1,554 & 2,038 \\
\hline $65-79$ & 0,630 & 0,079 & 64,077 & 1 & 0,000 & 1,877 & 1,609 & 2,190 \\
\hline$>79$ & 1,207 & 0,089 & 182,855 & 1 & 0,000 & 3,344 & 2,807 & 3,983 \\
\hline
\end{tabular}


Tabla 2 (continuación)

Variables individuales asociadas a la discapacidad*

\begin{tabular}{|c|c|c|c|c|c|c|c|c|}
\hline & \multirow{2}{*}{ B } & \multirow{2}{*}{ ET } & \multirow{2}{*}{ Wald } & \multirow{2}{*}{ gl } & \multirow{2}{*}{ Sig } & \multirow{2}{*}{ OR } & \multicolumn{2}{|c|}{ IC $95,0 \%$ OR } \\
\hline & & & & & & & Inferior & Superior \\
\hline \multicolumn{9}{|l|}{ Estado Civil } \\
\hline Soltero & & & 45,709 & 2 & 0,000 & 1 & & \\
\hline Casado & $-0,301$ & 0,053 & 32,475 & 1 & 0,000 & 0,740 & 0,667 & 0,821 \\
\hline Viudo-separado-divorciado & $-0,068$ & 0,061 & 1,245 & 1 & 0,265 & 0,935 & 0,830 & 1,053 \\
\hline \multicolumn{9}{|l|}{ Tipo ingresos } \\
\hline Por trabajo & & & & & & 1 & & \\
\hline Pensiones-subsidios-otros & 0,160 & 0,052 & 9,382 & 1 & 0,002 & 1,173 & 1,059 & 1,300 \\
\hline \multicolumn{9}{|l|}{ Número hijos anteriores } \\
\hline Ninguno & & & 10,053 & 4 & 0,040 & 1 & & \\
\hline 1 & $-0,090$ & 0,050 & 3,283 & 1 & 0,070 & 0,914 & 0,829 & 1,007 \\
\hline 2 & 0,055 & 0,054 & 1,067 & 1 & 0,302 & 1,057 & 0,952 & 1,174 \\
\hline 3 & 0,036 & 0,059 & 0,363 & 1 & 0,547 & 1,036 & 0,923 & 1,164 \\
\hline 4 o más & 0,048 & 0,049 & 0,961 & 1 & 0,327 & 1,050 & 0,953 & 1,156 \\
\hline \multicolumn{9}{|l|}{ Comunidad Autónoma } \\
\hline La Rioja & & & 179,261 & 17 & 0,000 & 1 & & \\
\hline Murcia & 1,057 & 0,210 & 25,237 & 1 & 0,000 & 2,877 & 1,905 & 4,345 \\
\hline Andalucía & 0,984 & 0,193 & 26,023 & 1 & 0,000 & 2,675 & 1,833 & 3,903 \\
\hline Galicia & 0,685 & 0,198 & 12,022 & 1 & 0,001 & 1,984 & 1,347 & 2,922 \\
\hline Ceuta y Melilla & 1,203 & 0,236 & 26,004 & 1 & 0,000 & 3,331 & 2,098 & 5,291 \\
\hline Castilla-León & 1,210 & 0,193 & 39,305 & 1 & 0,000 & 3,353 & 2,297 & 4,895 \\
\hline Extremadura & 0,425 & 0,214 & 3,921 & 1 & 0,048 & 1,529 & 1,004 & 2,328 \\
\hline Castilla-La Mancha & 0,761 & 0,200 & 14,540 & 1 & 0,000 & 2,141 & 1,448 & 3,167 \\
\hline Cataluña & 0,914 & 0,196 & 21,843 & 1 & 0,000 & 2,496 & 1,701 & 3,662 \\
\hline Asturias & 0,700 & 0,214 & 10,718 & 1 & 0,001 & 2,013 & 1,324 & 3,061 \\
\hline Valencia & 0,697 & 0,201 & 12,075 & 1 & 0,001 & 2,009 & 1,355 & 2,977 \\
\hline Cantabria & 1,006 & 0,224 & 20,215 & 1 & 0,000 & 2,735 & 1,764 & 4,240 \\
\hline Aragón & 1,056 & 0,205 & 26,430 & 1 & 0,000 & 2,875 & 1,922 & 4,300 \\
\hline Baleares & 0,488 & 0,226 & 4,662 & 1 & 0,031 & 1,629 & 1,046 & 2,537 \\
\hline Canarias & 0,497 & 0,211 & 5,525 & 1 & 0,019 & 1,644 & 1,086 & 2,488 \\
\hline Madrid & 0,847 & 0,207 & 16,758 & 1 & 0,000 & 2,333 & 1,555 & 3,499 \\
\hline Navarra & 0,568 & 0,233 & 5,927 & 1 & 0,015 & 1,764 & 1,117 & 2,786 \\
\hline País Vasco & 0,658 & 0,207 & 10,128 & 1 & 0,001 & 1,930 & 1,287 & 2,894 \\
\hline Constante & $-5,566$ & 0,220 & 640,691 & 1 & 0,000 & 0,004 & & \\
\hline
\end{tabular}

* Las variables (excepto CCAA de residencia) y estadísticos se corresponden con las variables del primer nivel (individual) de la regresión a dos niveles de la tabla 2.

ticos no cambian, estos mismos resultados no se presentan detallados en la tabla correspondiente a la regresión de dos niveles (tabla 2). En la tabla 3 en primer término se presenta el modelo correspondiente al nivel individual sin incluir ninguna de las variables del segundo nivel (modelo 0) y después se incluye el OR y la significación de las variables del segundo nivel cuando se realizó la regresión con cada una de ellas individualmente (modelos del 1 al 7). De los resultados cabe destacar que las variables individuales explican muy poco de la variabilidad entre CCAA (el porcentaje de la varianza explicada por el modelo 0 es o: 0,261 ) y 
Tabla 3

Modelos de regresión con las distintas variables del segundo nivel

\begin{tabular}{|c|c|c|c|c|c|}
\hline \multirow[t]{4}{*}{ Modelo 0} & Efectos aleatorios & $\sigma_{\mathrm{u} 0}$ & \multirow{3}{*}{\multicolumn{2}{|c|}{ IC $95 \%$}} & \multirow{4}{*}{$\frac{\text { p-valor }}{0,853}$} \\
\hline & CCAA (Nivel 2) & 0,261 & & & \\
\hline & Nivel 2 - Efectos fijos & OR & & & \\
\hline & $\%$ mayores de 65 años & 0,996 & 0,956 & 1,038 & \\
\hline \multirow[t]{4}{*}{ Modelo 1} & Efectos aleatorios & $\sigma_{\mathrm{u} 0}$ & \multirow{3}{*}{\multicolumn{2}{|c|}{ IC $95 \%$}} & \multirow[b]{3}{*}{ p-valor } \\
\hline & CCAA (Nivel 2) & 0,261 & & & \\
\hline & Nivel 2 - Efectos fijos & OR & & & \\
\hline & $\%$ familias monoparentales & 0,988 & 0,929 & 1,051 & 0,695 \\
\hline \multirow[t]{4}{*}{ Modelo 2} & Efectos aleatorios & $\sigma_{\mathrm{u} 0}$ & \multirow{3}{*}{\multicolumn{2}{|c|}{ IC $95 \%$}} & \multirow[b]{3}{*}{ p-valor } \\
\hline & CCAA (Nivel 2) & 0,259 & & & \\
\hline & Nivel 2 - Efectos fijos & OR & & & \\
\hline & Tasa de paro & 1,01 & 0,971 & 1,05 & 0,615 \\
\hline \multirow[t]{4}{*}{ Modelo 3} & Efectos aleatorios & $\sigma_{\mathrm{u} 0}$ & \multirow{3}{*}{\multicolumn{2}{|c|}{ IC $95 \%$}} & \multirow[b]{3}{*}{ p-valor } \\
\hline & CCAA (Nivel 2) & 0,259 & & & \\
\hline & Nivel 2 - Efectos fijos & OR & & & \\
\hline & $\%$ municipios $<10.000$ hab. & 0,996 & 0,991 & 1,002 & 0,195 \\
\hline \multirow[t]{4}{*}{ Modelo 4} & Efectos aleatorios & $\sigma_{\mathrm{u} 0}$ & \multirow{3}{*}{\multicolumn{2}{|c|}{ IC $95 \%$}} & \multirow[b]{3}{*}{ p-valor } \\
\hline & CCAA (Nivel 2) & 0,25 & & & \\
\hline & Nivel 2 - Efectos fijos & OR & & & \\
\hline & $\%$ hogares unipersonales & 0,945 & 0,883 & 1,011 & 0,094 \\
\hline \multirow[t]{4}{*}{ Modelo 5} & Efectos aleatorios & $\sigma_{\mathrm{u} 0}$ & \multirow{3}{*}{\multicolumn{2}{|c|}{ IC $95 \%$}} & \multirow[b]{3}{*}{ p-valor } \\
\hline & CCAA (Nivel 2) & 0,243 & & & \\
\hline & Nivel 2 - Efectos fijos & OR & & & \\
\hline & Ratio plazas larga estancia / 65 años & 1,006 & 0,89 & 1,137 & 0,921 \\
\hline \multirow[t]{4}{*}{ Modelo 6} & Efectos aleatorios & $\sigma_{\mathrm{u} 0}$ & \multirow{3}{*}{\multicolumn{2}{|c|}{ IC $95 \%$}} & \multirow[b]{3}{*}{$p$-valor } \\
\hline & CCAA (Nivel 2) & 0,261 & & & \\
\hline & Nivel 2 - Efectos fijos & OR & & & \\
\hline & Coches matriculados por 100 hab. & 1,007 & 0,905 & 1,12 & 0,897 \\
\hline \multirow[t]{2}{*}{ Modelo 7} & Efectos aleatorios & $\sigma_{\mathrm{u} 0}$ & & & \\
\hline & CCAA (Nivel 2) & 0,261 & & & \\
\hline
\end{tabular}

que tampoco ninguna de las variables del segundo nivel colabora en mejorar esta capacidad explicativa, ya que ninguna de ellas resulta ser significativa.

\section{DISCUSIÓN}

Como otros trabajos han puesto de manifiesto $^{7,13}$, la prevalencia de discapacidad entre los distintos territorios y estimaciones tiene una variabilidad importante. Las diferencias se reducen al estandarizar por edad, pero no desaparecen,. Es decir, que el distinto grado de envejecimiento de la población de estas comunidades no es suficiente para explicar las diferencias entre ellas.

Para analizar qué factores podían ayudar a explicar esta variabilidad se han realizado dos análisis consecutivos encaminados a tratar de identificar las fuentes de variación desde la perspectiva individual y del entorno. Además, previamente se verificó que la muestra de población que respondió al cuestionario de salud saludis (cuyos datos eran mucho más ricos que los de la muestra de discapacidad tododis), podía ser utilizada como una aproximación válida y representativa de la población general. Para ello se compararon las estimaciones de la preva- 
lencia de discapacidad por CCAA obtenidas de las dos muestras y los resultados mostraron que eran parecidas. Este resultado es muy interesante por que corrobora que la variabilidad entre CCAA se mantiene al usar dos fuentes de datos distintas. Por otra parte, al ser las estimaciones aceptablemente consistentes entre sí se han podido utilizar ambas en función de la estrategia de análisis prevista.

Tal como era previsible, del estudio de los factores asociados a la discapacidad destaca que padecer discapacidad se asocia a algunas características individuales de las personas, como el sexo (hombre), edad mayor de 79 años, estado civil (soltero) y situación socioeconómica (sin estudios, sin trabajo). Además, las personas con discapacidad presentan una peor situación de salud en general (con mayor número de enfermedades crónicas, consumo de medicamentos y peor percepción del propio estado de salud) y una relación con el entorno más limitada (dificultades con la vivienda, con el uso del coche y del transporte público). En general, los factores identificados son consistentes con los publicados en la literatura científica, como son la edad, la mayor morbilidad crónica, nivel educativo bajo y peor percepción de salud ${ }^{2,14-17}$, con una relevante excepción, el sexo.

Habitualmente se considera que existe una mayor discapacidad en el sexo femenino. También en todos los análisis descriptivos efectuados con los datos de este estudio la población femenina padecía mayor discapacidad, tanto al analizarla en su conjunto como por tipos de discapacidad y por gravedad. Por este motivo parecerían paradójicos los resultados obtenidos de la regresión múltiple, que muestran un efecto protector, es decir de menor riesgo de discapacidad en las mujeres. Una vez descartados posibles errores en la codificación y análisis de los datos se procedió a un conjunto de análisis complementarios para tratar de buscar la explicación más plausible a este resultado. Entre los más relevantes destaca que al utilizar en la regresión solo la variable sexo, el femenino se asocia a una mayor discapacidad, no obstante, al ir introduciendo otras variables en la regresión cambiaba el sentido de la asociación. Por este motivo pensamos que esta asociación "paradójica" podría ser debida al efecto de alguna interacción entre las co-variables que sin embargo no se pudo identificar.

Estos resultados concuerdan con otras observaciones reflejadas en la literatura, en las que también se obtiene este resultado aparentemente paradójico. En los análisis descriptivos, numerosos estudios muestran que las mujeres presentan una mayor discapacidad, no obstante, al ajustar por otras covariables en muchos casos desaparece la significación estadística de la asociación ${ }^{15}$, 17-19. Un estudio realizado específicamente para dilucidar el efecto del sexo en el proceso de discapacidad obtiene unos resultados que justificarían nuestra hipótesis, es decir, que no existe efecto directo del sexo sobre la discapacidad si no que la relación está mediatizada por otras co-variables (como la edad y algunas patologías específicas) y puede ser distinta según la gravedad de la discapacidad ${ }^{20}$. Esto se vería reforzado por el hecho de que el proceso de discapacitación parece ser el mismo en los dos sexos, aunque con edades de inicio más tempranas en las mujeres ${ }^{21}$.

Por este motivo creemos que es importante mostrar este resultado para que pueda ser tenido en consideración en futuros análisis con los datos de discapacidad. Dependiendo del objetivo del estudio las estrategias de análisis para hacer frente a esta paradoja pueden ser distintas, como la de excluir la variable sexo en los estudios de factores de riesgo, puesto que otras variables en el modelo (edad, estado civil, etc.) neutralizan su efecto ${ }^{7} \mathrm{o}$, al contrario, diferenciar por sexo todos los cálculos ${ }^{2,16}$, lo que evitaría estos problemas de interacción aunque no permitiría comparar tan fácil- 
mente entre los dos sexos. No obstante, no hay que olvidar que en algunos análisis el sexo es probablemente una variable más descriptiva de la estructura demográfica que un factor determinante de la discapacidad, lo cual, a nuestro entender, justificaría su inclusión en determinados estudios, como sería el caso de la variabilidad geográfica.

De los resultados de esta regresión destaca también la gradación del riesgo de discapacidad asociada a la comunidad autónoma de residencia, de manera que Castilla-León (prevalencia más alta) tiene un riesgo más de tres veces mayor que La Rioja (la más baja). Este resultado pone en evidencia que el lugar de residencia es un factor explicativo muy importante, ya que se mantiene a pesar de ajustar por el resto de variables individuales consideradas clásicamente determinantes de la salud.

Las diferencias geográficas en la prevalencia de discapacidad ya se han puesto en evidencia en otros estudios y generalmente se relacionan con factores socioeconómicos o educativos ${ }^{2,17-18,22}$. Además de los factores mencionados a nivel individual y puesto que la comunidad autónoma de residencia aparecía como una variable significativa, en el presente trabajo se consideró adecuado incorporar variables que caracterizaran las CCAA (en relación con las condiciones económicas, demográficas y de recursos sociosanitarios) en una regresión de dos niveles. Este tipo de análisis considera de manera simultánea la relación de la discapacidad con las variables que caracterizan a los individuos y a los grupos (en este caso las relativas al lugar de residencia). Es decir, tiene en cuenta que la probabilidad de discapacidad, aún teniendo las mismas características individuales, podría ser distinta en función de las características del lugar de residencia, si éstas resultaran significativas en el modelo. Sin embargo, en los resultados de la regresión con dos niveles no se identificó ningún factor relativo a la comunidad que fuera estadísticamente sig- nificativo. A pesar de ello, puesto que las variables individuales por si mismas solo explican un porcentaje muy pequeño de la variación entre CCAA, cabe pensar que otros factores distintos a los analizados, pero que difieran entre las CCAA, podrían proporcionar una mejor explicación de esta variabilidad. Además, habría que considerar que el grado de co-discapacidad o del nivel de gravedad de las discapacidades tenga una distribución diferente por CCAA. Esto implicaría estudiar más a fondo las características del tipo y gravedad de las discapacidades por comunidad, probablemente a partir de métodos clínico-epidemiológicos y fuentes de información más específicas. Será necesario seguir esta línea de investigación para poder clarificar esta cuestión que tiene importantes consecuencias desde el punto de vista sanitario ${ }^{8}$.

Este trabajo presenta algunas limitaciones debidas principalmente a la procedencia de los datos de dos muestras y al hecho que se trata de un estudio transversal, de forma que la relación entre la discapacidad $\mathrm{y}$ algunas variables que resultaron significativas (dificultades en el transporte, actividad laboral, etc.) seguramente pone más de relieve las consecuencias que las causas de la discapacidad. Los resultados de la prueba de consistencia de los datos realizada al comparar las estimaciones de prevalencia resultantes de las dos muestras (la procedente del cuestionario de salud con respecto a la muestra del cuestionario de discapacidad), nos parecen aceptables puesto que se observan diferencias únicamente en el caso de Aragón, hecho que habrá que tener en cuenta al interpretar los datos de esa comunidad.

Por otra parte, la pertinencia de las variables usadas para caracterizar las CCAA en el segundo nivel de la regresión puede ser cuestionable, dado que fueron seleccionadas de forma un tanto oportunista a partir de las estadísticas disponibles, además de los problemas de calidad y comparabilidad de 
la información relativa a los recursos y servicios asistenciales para personas mayores y/o discapacitadas. Este hecho podría ayudar a explicar la falta de significación obtenida en los resultados y sin duda debería estimular a una mayor investigación para la mejora de la información y los métodos que permitan profundizar en los modelos de análisis geográfico.

En conclusión, cabria resaltar que la variabilidad geográfica de la discapacidad entre comunidades autónomas es muy importante y que no se explica por la distinta distribución de los factores individuales considerados tradicionalmente determinantes de la salud. El sexo femenino muestra una asociación "paradójica" con la discapacidad, de forma que en los análisis bivariados se asocia a la discapacidad pero al considerar otras variables como la edad o las condiciones de vida esta relación desaparece. No se han identificado variables caracterizadoras de las CCAA que expliquen la variabilidad de la discapacidad entre ellas. En parte puede ser debido a la poca calidad o pertinencia de la información y a la perspectiva ecológica del estudio. Es necesario profundizar más en el conocimiento de la distribución geográfica de los tipos y gravedad de la discapacidad así como de los factores del entorno que se asocian a esta distribución.

\section{AGRADECIMIENTOS}

Al resto de personas participantes en el proyecto por sus aportaciones: Josep $\mathrm{M}^{\mathrm{a}}$ Argimón, $\mathbf{M}^{\mathrm{a}}$ Aran Barés, Anna Puigdefàbregas, Cecilia Ruíz y M Mar Torné. A Gloria Ribas por sus comentarios.

\section{BIBLIOGRAFÍA}

1. Béland F, Zunzunegui MV. La salud y las incapacidades funcionales. Elaboración de un modelo causal. Rev Gerontol. 1995; 5: 259-273.
2. Valderrama-Gama E, Damián J, Ruigómez A, Martin-Moreno JM. Chronic disease, functional status and self-ascribed causes of disabilities among non institutionalized older people in Spain. J Gerontol A Biol Sci Med Sci. 2002; 57A(11): M716-M721.

3. Escobar MA, Puga D, Martín MT. Asociaciones en la red social y la discapacidad al comienzo de la vejez en las ciudades de Madrid y Barcelona en 2005. Rev Esp Salud Pública. 2008; 82(6): 637651.

4. Verbrugge LM, Jette AM. The disablement process. Soc Sci Med. 1994; 38(1): 1-14.

5. Pluijm SMF, Bardage C, Nikula S, Blumstein T, Jylha M, Minicuci N, Zunzunegui V, Pedersen NL, Deeg D. A harmonized measure of activities of daily living was a reliable and valid instrument for comparing disability in older people across countries. J Clin Epidemiol. 2005; 58: 1015-23.

6. Gispert R, Clot-Razquin G, Rivero T, Freitas A, Ruíz-Ramos M, Ruíz-Luque C, Busquets E, Argimón JM. El perfil de la dependencia en España: un análisis de la encuesta de discapacidades de 1999. Rev Esp Salud Pública. 2008; 82(6): 653-665.

7. INE. Encuesta sobre discapacidades, deficiencias y estado de salud. Año 1999. Madrid: INE, febrero de 1999.

8. Gispert R. Hacia una mayor adecuación y equidad del programa de ayudas a las personas con dependencia. Rev Esp Salud Pública. 2009; 83(3): 355-9.

9. Gispert R, Ruíz-Ramos M, Barés MA, Viciana F, Clot-Razquin G. Diferencias en la esperanza de vida libre de discapacidad por sexo y Comunidades Autónomas en España. Rev Esp Salud Pública. $2007 ;$ 81(2): 155-165.

10. Instituto Nacional de Estadística. INEBASE. Disponible en: www.ine.es/inebmenu/indice.htm

11. Ministerio de Trabajo y Asuntos Sociales. Imserso. Las personas mayores en España, Informe de 2002. Disponible en: www.imsersomayores.csic. es/estadisticas/informemayores/informe2002/index.html

12. R 2.4.1- A language and environment, 2006. Disponible en. http://www.cran.r-project.org

13. Abellán García A, Palacios Ramos E, Esparza Catalán C. Diferentes estimaciones de la discapacidad y la dependencia en España. Informes Por- 
tal Mayores núm 56. Disponible en: www.imsersomayores.csic.es/documentacion/estudiosyresultados/informes/index.html

14. Beland F, Zunzunegui MV. Predictors of functional status in older people living at home. Age Ageing. 1999; 28: 153-9.

15. Reynolds S, Silverstein M. Observing the onset of disability in older adults. Soc Sci Med. 2003; 57 : 1875-1889.

16. Graciani A, Banegas JR, López-García, Rodriguez-Artalejo F. Prevalence of disability and associated social and health-related factors among the elderly in Spain: a population-based study. Maturitas. 2004; 48: 381-92.

17. Matthews R, Smith L, Hancock R, Jagger C, Spiers N. Socioeconomic factors associated with the onset of disability in older age: a longitudinal study of people aged 75 years and over. Soc Sci Med. 2005; 61: 1567-75.
18. Raina P, Dukeshire S, Lindsay J, Chambers L. Chronic Conditions and disabilities among seniors: an analysis of population-based health and activity limitation surveys. Ann Epidemiol. 1998; 8: 402-9.

19. Dunlop D, Manheim L, Sohn M-W, Liu X, Chang RW. Incidence of functional limitation in older adults: the impact of gender, race, and chronic conditions. Arch Phys Med Rehabil. 2002; 83: 964-71.

20. Wray L, Blaum C. Explaining the role of sex on disability: a population-based study. Gerontologist. 2001; 41(4): 499-510.

21. Jagger C, Antony A, Spiers N, Clarke M. Patterns of onset of disability in activities of daily living with age. J Am Geriatric Soc. 2001; 49(4): 404-9.

22. Mackenbach JP, Borsboom GJJM, Nusselder WJ, 